\section{Caracterização dos medicamentos prescritos aos idosos na Estratégia Saúde da Família}

\author{
Characterization of drugs prescribed to the elderly \\ in the Family Health Strategy
}

\author{
${ }_{1}$ Faculdade de Medicina de \\ Marília, Marília, Brasil. \\ 2 Programa de Pós-gradu- \\ ação em Ciências da Saúde, \\ Universidade Federal de São \\ Paulo, São Paulo, Brasil. \\ Correspondência \\ M. J. S. Marin \\ Faculdade de Medicina de \\ Marília. \\ Av. Brigadeiro Eduardo \\ Gomes 1886, Marília, SP \\ 17514-000, Brasil. \\ marnadia@terra.com.br
}

\begin{abstract}
Considering that the elderly are heavy consumers of medications, with significant consequences for their living conditions, the current study was intended to analyze the characteristics of such drug prescription, using the indicators proposed by the World Health Organization (WHO), and to characterize the drug classes prescribed to them in four units under the Family Health Strategy (FHS) in Marília, São Paulo State, Brazil. One family health unit (FHU) was selected by region of the city, and 100 patient charts were checked in FHU-A; 86 in B, 99 in C, and 97 in $D$. According to WHO drug prescription criteria, the results were similar to the recommended patterns except for drug prescription by generic name. In addition, there were significant differences between the family health units. The most frequently prescribed drug classes were for cardiovascular disorders. The health professionals showed a concern for adjusting drug prescription to rational-use criteria. However, there is a need for understanding the differences in prescription between units and a search for consensus, aimed at rational drug use.
\end{abstract}

Drug Prescriptions; Health of the Elderly; Family Health Program
Camila Alves Paes de Oliveira ${ }^{1}$

Maria José Sanches Marin 1,2

Milton Marchioli 1

Bruno Henrique Magalhães Pizoletto 1

Rafael Varella dos Santos 1

\section{Introdução}

O envelhecimento sempre foi motivo de preocupação para o homem. No entanto, nas últimas décadas, com o crescente aumento da população idosa, tem-se acentuado a busca para compreender esse processo, evidenciar seus problemas e estabelecer propostas para se viver melhor esta etapa da vida.

A população idosa brasileira, em 2002, perfazia um total de 14,1 milhões de pessoas. Projetase, para 2025, um total de 33,4 milhões, e entre 1950 e 2025 a população idosa crescerá 16 vezes contra cinco vezes a população total 1 . Destacase na população idosa o aumento do número de pessoas com 80 anos ou mais, sendo essa a faixa etária de maior crescimento, tanto nos países desenvolvidos quanto nos países em desenvolvimento 2 .

As pessoas idosas apresentam características específicas do ponto de vista fisiológico, psicológico e social, decorrentes das perdas que ocorrem ao longo da vida e que as tornam vulneráveis às alterações no estado de saúde ${ }^{3}$.

As múltiplas alterações apresentadas pelos idosos fazem com que eles sejam consumidores de grande número de medicamentos. Considerase que a maioria utiliza mais de um medicamento periodicamente e, quando hospitalizados, recebem de 8 a 15 4,5. Nos Estados Unidos, estima-se que de $25 \%$ a $32 \%$ de toda a medicação prescrita seja para uso dos idosos, os quais representam 
$12 \%$ da população ${ }^{6}$. No Japão, em 1995, a população com 65 anos ou mais era de 14,5\%, com consumo de $30,6 \%$ de todos os medicamentos prescritos 7 . Os efeitos colaterais das drogas são duas vezes e meia mais freqüentes nos idosos do que na população de outra faixa etária ${ }^{6}$.

No Brasil, estudo baseado na Pesquisa Nacional por Amostra de Domicílios (PNAD) aponta que $50 \%$ dos idosos têm renda pessoal menor que um salário mínimo e o gasto médio mensal com medicamentos compromete aproximadamente um quarto dessa renda 8 .

Esse consumo de múltiplos medicamentos, comum entre os idosos, embora necessário em muitas ocasiões, quando inadequado pode desencadear complicações sérias ao levar a situações de polifarmácia ("uso de pelo menos uma droga desnecessária no rol de prescrições supostamente necessárias") e de iatrogenia ("efeito patogênico de uma droga ou interação de várias drogas") 9 (p. 4).

A polifarmácia favorece o sinergismo e o antagonismo não desejado, o descumprimento das prescrições de medicamentos não essenciais e gastos excedentes com aqueles de uso supérfluo ${ }^{10}$. Além disso, como muitas vezes o tratamento é instituído por vários especialistas que acrescentam drogas em vez de suspenderem ou modificarem a posologia, é comum encontrar prescrições de doses e indicações inadequadas, interações e associações medicamentosas, redundância, além do uso de medicamentos sem valor terapêutico ${ }^{11}$.

Visando à maior adequação no uso de medicamentos, políticas vêm sendo propostas. Em nível nacional, a Política Nacional de Medicamentos tem-se constituído no principal instrumento para a orientação das ações de saúde relacionadas ao uso de medicamentos, sendo o principal objetivo garantir a necessária segurança, eficácia e qualidade dos medicamentos, a promoção do uso racional e o acesso da população àqueles considerados essenciais 12 .

Entre suas prioridades, está o uso racional de medicamentos que compreende a prescrição apropriada; a disponibilidade oportuna e a preços acessíveis; a dispensação em condições adequadas; e o consumo nas doses indicadas, nos intervalos definidos e no período de tempo indicado, de medicamentos eficazes, seguros e de qualidade 12.

A Organização Mundial da Saúde (OMS) desenvolveu indicadores de qualidade e quantidade de medicamentos para estimular o uso racional. São os principais indicadores de uso de medicamentos: média de medicamentos por prescrição - objetiva medir o grau de polimedicação do paciente; porcentagem de medicamentos prescritos pelo nome genérico - visando a propiciar o controle dos custos de medicamentos no serviço de saúde; porcentagem de medicamentos prescritos da lista de medicamentos essenciais - cujo objetivo é garantir o tratamento para as principais patologias da população; porcentagem de antibióticos prescritos - com a finalidade de controlar o seu uso e evitar resistências bacterianas e; porcentagem de injetáveis prescritos - pela maior possibilidade de conseqüências quando prescritos ou aplicados de forma inadequada 13 .

Diante da intensidade e da complexidade dos problemas apresentados pelos idosos no que se refere ao uso de medicamentos, bem como das políticas que vêm sendo propostas pelo Ministério da Saúde com a finalidade de promover o uso racional, propõe-se para o presente estudo analisar as medicações prescritas aos idosos usuários dos serviços em diferentes unidades de saúde da família (USF) do Município de Marília, São Paulo, Brasil, utilizando os indicadores de prescrição de medicamentos propostos pela OMS e, também, caracterizar as classes farmacológicas dos medicamentos prescritos aos idosos nas diferentes USF.

\section{Metodologia}

O estudo foi realizado nas USF do Município de Marília, cuja implantação ocorreu no período de 1998 a 2005, contando-se atualmente com 28 unidades localizadas em áreas onde a população apresenta maior carência sócio-econômica e, conseqüentemente, menor possibilidade de acesso aos serviços de saúde e maior exposição aos riscos de adoecer e morrer. A população estimada da cidade de Marília é de 218.113 habitantes (Instituto Brasileiro de Geografia e Estatística.Cidades@: Marília - SP; http://www.ibge. gov.br/cidadesat/topwindow.htm?1, acessado em 27/Nov/2007), tendo aproximadamente 84 mil habitantes atendidos pela Estratégia Saúde da Família (ESF). Dentre os atendidos, 14.815 são idosos.

Para a coleta de dados foi sorteada uma USF por região da cidade (norte, sul, leste, oeste), mas tais regiões apresentam oito, nove, quatro e sete unidades, respectivamente. As quatro USF selecionadas foram denominadas de A, B, C e D, com a finalidade de se garantir $\mathrm{o}$ anonimato.

A amostra estudada foi de 382 pessoas idosas e definida pelo cálculo de tamanho de amostra aleatória simples para erro amostral tolerável igual a $5 \% 14$. A amostra foi proporcional ao tamanho da população de idosos de cada USF selecionada, sendo verificados 100 prontuários na USF A; 86 na $B, 99$ na $C$ e 97 na $D$, as quais 
contam com 360, 309, 356, 348 idosos, respectivamente.

A coleta de dados foi realizada por meio da verificação dos medicamentos prescritos aos idosos nos prontuários, no período de 10 de setembro de 2005 a 30 de agosto de 2006 e seguindo um roteiro, previamente estabelecido, contendo sexo, idade, medicamentos prescritos em cada consulta com sua respectiva dose e via de administração. Os prontuários foram selecionados aleatoriamente pelos pesquisadores, com base nos cadastros dos agentes comunitários de saúde (ACS).

Para a análise dos dados, foram considerados os indicadores de prescrição de medicamentos propostos pela OMS: número de medicamentos prescritos por consulta médica; número de medicamentos prescritos pelo nome genérico; número de consultas em que foram prescritos antibióticos; número de consultas em que foi prescrito pelo menos um injetável; número de medicamentos prescritos que figuram na lista de medicamentos padronizados pelo município. Para identificação dos medicamentos padronizados e não padronizados, foi utilizada a relação de medicamentos essenciais selecionados pela Secretaria Municipal de Saúde de Marília 15.

Os medicamentos foram classificados de acordo com Anatomical Therapeutic Chemical Code (ATC) adotada pela OMS 13. Nessa classificação, os fármacos são divididos de acordo com o grupo anatômico, ou sistema em que atuam e suas propriedades químicas, terapêuticas e farmacológicas. Para identificar as substâncias mediante seus nomes comerciais, empregou-se o DEF 2005/06: Dicionário de Especialidades Farmacêuticas 16 .

O estudo contou com a autorização da Secretaria Municipal de Saúde de Marília e foi aprovado pelo Comitê de Ética e Pesquisa com Seres Humanos da Faculdade de Medicina de Marília.

Os dados são apresentados na forma de tabelas, em freqüência percentual simples, seguida de análise descritiva e comparativa com estudos realizados em outras cidades e regiões.

\section{Resultados}

As unidades selecionadas apresentam diferenciações quanto ao perfil sócio-econômico da população, cujas características principais encontram-se no Tabela 1, em que se observa que na USF A, apenas $5,8 \%$ da população têm algum tipo de plano de saúde, enquanto na população da USF C, 44,3\% têm plano de saúde. Destacase, ainda, na USF B que quase $14 \%$ residem em casa de madeira ou de material reaproveitado, o
Tabela 1

Distribuição percentual das principais características sócio-econômicas da população das unidades de saúde da família (USF) A, B, C e D *. Marília, São Paulo, Brasil, 2007.

\begin{tabular}{lcccc}
\hline Características & \multicolumn{3}{c}{ USF } \\
& A (\%) & B (\%) & C (\%) & D (\%) \\
\hline Plano de saúde & 5,8 & 6,3 & 44,23 & 16,4 \\
Abastecimento de água & 99,1 & 100,0 & 99,0 & 98,8 \\
Esgoto & 88,4 & 100,0 & 99,8 & 100,0 \\
Coleta de lixo & 98,5 & 100,0 & 99,6 & 100,0 \\
Luz elétrica & 99,0 & 99,9 & 97,7 & 99,2 \\
Casa de alvenaria & 97,4 & 85,9 & 99,0 & 100,0 \\
\hline
\end{tabular}

* Dados obtidos do Sistema de Informação da Atenção Básica (SIAB) 40.

que não acontece nas demais unidades em que quase a totalidade das pessoas reside em casa de alvenaria.

A Tabela 2 apresenta a distribuição dos idosos de acordo com a idade e sexo, nas unidades estudadas (A, B, C e D). Observa-se maior porcentagem de idosos na faixa etária dos 60-69 anos, em todas as USF, em um total de 203 idosos $(53,1 \%)$. O sexo feminino também foi predominante, 224 $(58,6 \%)$ do total de idosos.

Quanto às médias de consultas por idoso no período de um ano nas unidades estudadas, foi possível constatar que os 382 idosos realizaram 1.479 consultas médicas, com uma média de 3,8 consultas/idoso/ano. A USF A apresentou uma média de 3,3 consultas/idoso/ano; a USF B, a média de 3,0 consultas/idoso/ano e a USF D de 3,8 consultas/idoso/ano, enquanto na USF C essa média foi de 4,7 consultas/idoso/ano.

Os indicadores de prescrição medicamentosa propostos pela OMS, quando verificados nas unidades estudadas, conforme descrito na Tabela 3, apresentaram-se quanto ao número de medicamentos prescritos por consulta, da seguinte forma: na USF A, em 71 (21,5\%) das consultas não foram prescritos medicamentos e em 10 (3\%) foram prescritos cinco ou mais, na USF B, em $20(7,6 \%)$ não foram prescritos medicamentos e em 57 (21,6\%) foram prescritos cinco ou mais. Na USF C foram prescritos antibióticos em $48(10,1 \%)$ das consultas, e nas demais unidades foram prescritos antibióticos em torno de $4 \%$ a $5 \%$ das consultas. A USF D foi aquela que apresentou menores índices de prescrição de medicamentos pelo nome genérico: $85,5 \%$. Nas USF C e D, os medicamentos injetáveis foram prescritos pelo menos uma vez em 12 (2,5\%) e $15(3,7 \%)$ das consultas, respectivamente. O número de prescrições de medicamentos não 
Tabela 2

Distribuição dos idosos de acordo com faixa etária e sexo nas unidades de saúde da família (USF) A, B, C e D. Marília, São Paulo, Brasil, 2007.

\begin{tabular}{|c|c|c|c|c|c|c|c|c|c|c|}
\hline \multirow[t]{3}{*}{ Idade (anos) } & \multicolumn{8}{|c|}{ USF } & \multicolumn{2}{|c|}{ Total } \\
\hline & \multicolumn{2}{|c|}{ A } & \multicolumn{2}{|c|}{ B } & \multicolumn{2}{|c|}{ C } & \multicolumn{2}{|c|}{ D } & & \\
\hline & $\begin{array}{c}\text { Masculino } \\
\text { n (\%) }\end{array}$ & $\begin{array}{c}\text { Feminino } \\
\text { n (\%) }\end{array}$ & $\begin{array}{c}\text { Masculino } \\
\text { n (\%) }\end{array}$ & $\begin{array}{c}\text { Feminino } \\
\text { n (\%) }\end{array}$ & $\begin{array}{c}\text { Masculino } \\
\text { n (\%) }\end{array}$ & $\begin{array}{c}\text { Feminino } \\
n(\%)\end{array}$ & $\begin{array}{c}\text { Masculino } \\
\text { n (\%) }\end{array}$ & $\begin{array}{c}\text { Feminino } \\
\text { n (\%) }\end{array}$ & $\begin{array}{c}\text { Masculino } \\
\text { n (\%) }\end{array}$ & $\begin{array}{c}\text { Feminino } \\
\text { n (\%) }\end{array}$ \\
\hline $60-69$ & $29(29,0)$ & $34(34,0)$ & $16(18,6)$ & $20(23,3)$ & $18(18,2)$ & $31(31,3)$ & $23(23,7)$ & $32(33,0)$ & $86(42,4)$ & $117(57,6)$ \\
\hline $70-79$ & $12(12,0)$ & $16(16,0)$ & $9(10,5)$ & $20(23,3)$ & $18(18,2)$ & $14(14,1)$ & $11(11,3)$ & $21(21,6)$ & $50(41,3)$ & $71(58,7)$ \\
\hline 80 ou mais & $5(5,0)$ & $4(4,0)$ & $7(8,1)$ & $14(16,3)$ & $7(7,1)$ & $11(11,1)$ & $3(3,1)$ & $7(7,2)$ & $22(37,9)$ & $36(62,1)$ \\
\hline Total & $46(46,0)$ & $54(54,0)$ & $32(37,2)$ & $54(62,8)$ & $43(43,4)$ & $56(56,6)$ & $37(38,1)$ & $60(61,9)$ & $158(41,4)$ & $224(58,6)$ \\
\hline
\end{tabular}

Tabela 3

Distribuição dos indicadores de prescrição medicamentosa de acordo com as unidades de saúde da família (USF) A, B, C e D. Marília, São Paulo, Brasil, 2007.

\begin{tabular}{|c|c|c|c|c|}
\hline \multirow[t]{4}{*}{ Indicadores de prescrição } & \multicolumn{4}{|c|}{ USF } \\
\hline & A & B & C & D \\
\hline & (100 prontuários) & (86 prontuários) & (99 prontuários) & (97 prontuários) \\
\hline & n (\%) & $n(\%)$ & n (\%) & n (\%) \\
\hline \multicolumn{5}{|l|}{ Número de medicamentos/consulta } \\
\hline 0 & $71(21,5)$ & $20(7,6)$ & $53(11,2)$ & $57(13,9)$ \\
\hline 1 & $105(31,7)$ & $44(16,6)$ & $154(32,5)$ & $105(25,6)$ \\
\hline 2 & $87(26,3)$ & $76(28,8)$ & $83(17,5)$ & $76(18,5)$ \\
\hline 3 & $37(11,2)$ & $39(14,8)$ & $85(17,9)$ & $70(17,0)$ \\
\hline 4 & $21(6,3)$ & $28(10,6)$ & $59(12,4)$ & $38(9,3)$ \\
\hline 5 ou mais & $10(3,0)$ & $57(21,6)$ & $40(8,4)$ & $64(15,6)$ \\
\hline Total & $331(100,0)$ & $264(100,0)$ & $474(100,0)$ & $410(100,0)$ \\
\hline \multicolumn{5}{|l|}{ Número de antibióticos/consulta } \\
\hline 0 & $313(94,6)$ & $252(95,5)$ & $425(89,7)$ & $388(94,6)$ \\
\hline 1 & $17(5,1)$ & $12(4,5)$ & $48(10,1)$ & $22(5,4)$ \\
\hline 2 & $1(0,3)$ & $0(0,0)$ & $1(0,2)$ & $0(0,0)$ \\
\hline 3 ou mais & $0(0,0)$ & $0(0,0)$ & $0(0,0)$ & $0(0,0)$ \\
\hline Total & $331(100,0)$ & $264(100,0)$ & $474(100,0)$ & $410(100,0)$ \\
\hline \multicolumn{5}{|l|}{ Número de injetáveis/consulta } \\
\hline 0 & $327(98,8)$ & $264(100,0)$ & $461(97,3)$ & $395(96,3)$ \\
\hline 1 & $4(1,2)$ & $0(0,0)$ & $12(2,5)$ & $15(3,6)$ \\
\hline 2 & $0(0,0)$ & $0(0,0)$ & $1(0,2)$ & $0(0,0)$ \\
\hline 3 ou mais & $0(0,0)$ & $0(0,0)$ & $0(0,0)$ & $0(0,0)$ \\
\hline Total & $331(100,0)$ & $264(100,0)$ & $474(100,0)$ & $410(100,0)$ \\
\hline \multicolumn{5}{|l|}{ Número de medicamentos prescritos } \\
\hline \multicolumn{5}{|l|}{ pela Secretaria da Saúde } \\
\hline $\begin{array}{l}\text { Número de medicamentos } \\
\text { prescritos com nome genérico }\end{array}$ & $271(93,8)$ & $305(88,4)$ & $471(87,7)$ & $479(85,5)$ \\
\hline
\end{tabular}


constantes da lista de selecionados pela Secretaria Municipal de Saúde de Marília foi menor na unidade D: $77 \%$.

Entre os prontuários analisados constatouse que os medicamentos mais prescritos foram aqueles do sistema cardiovascular, sendo $28,7 \%$; $30,5 \% ; 24,8 \%$ e $29,1 \%$, respectivamente nas USF A, B, C e D, com uma média de $27,9 \%$. Na seqüência encontram-se os medicamentos de uso sistêmico, cuja freqüência foi de $32,1 \%$ na USF A, $22,3 \% \%$ na USF B, $27,7 \%$ na USF C e de $24,5 \%$ na USF D. Os medicamentos que atuam no sistema digestório e metabolismo representam 11,6\% na USF A, $14,2 \%$ na USF B, $17,8 \%$ na USF C e $16,3 \%$ na USF D. Os medicamentos relacionados ao sistema nervoso representam 11,\%, 10,2\%, 10,5\% e $13,4 \%$ dos medicamentos prescritos nas USF A, B, C e D (Tabela 4).

\begin{tabular}{|c|c|c|c|c|c|}
\hline \multirow[t]{3}{*}{ Classes de medicamentos } & \multicolumn{5}{|c|}{ USF } \\
\hline & $A$ & B & $\mathrm{C}$ & $\mathrm{D}$ & Total \\
\hline & n (\%) & n (\%) & n (\%) & n (\%) & n (\%) \\
\hline \multicolumn{6}{|l|}{ Sistema cardiovascular } \\
\hline Anti-hipertensivo & $43(14,9)$ & $57(16,5)$ & $79(14,7)$ & $84(15,0)$ & $263(15,2)$ \\
\hline Diurético & $36(12,5)$ & $39(11,3)$ & $44(8,2)$ & $67(12,0)$ & $186(10,7)$ \\
\hline Cardiotônico & $3(1,0)$ & $4(1,2)$ & $5(0,9)$ & $8(1,4)$ & $20(1,2)$ \\
\hline Antianginoso & $1(0,3)$ & $1(0,3)$ & $2(0,4)$ & $2(0,4)$ & $6(0,3)$ \\
\hline Antiarrítmico & $0(0,0)$ & $4(1,2)$ & $3(0,6)$ & $2(0,4)$ & $9(0,5)$ \\
\hline Subtotal & $83(28,7)$ & $105(30,5)$ & $133(24,8)$ & $163(29,2)$ & $484(27,9)$ \\
\hline \multicolumn{6}{|l|}{ Sistema nervoso central } \\
\hline Hipnóticos, ansiolítico & $9(3,1)$ & $10(2,9)$ & $16(3,0)$ & $26(4,6)$ & $61(3,5)$ \\
\hline Antidepressivos e antimaníaco & $11(3,8)$ & $17(4,9)$ & $26(4,9)$ & $20(3,6)$ & $74(4,3)$ \\
\hline Vasodilatador & $3(1,0)$ & $4(1,2)$ & $3(0,6)$ & $7(1,3)$ & $17(1,0)$ \\
\hline Anticonvulsivante & $4(1,4)$ & $2(0,6)$ & $3(0,6)$ & $2(0,4)$ & $11(0,6)$ \\
\hline Antipsicótico & $3(1,0)$ & $2(0,6)$ & $2(0,4)$ & $10(1,8)$ & $17(1,0)$ \\
\hline Antiparkinsoniano & $2(0,7)$ & $0(0,0)$ & $3(0,6)$ & $7(1,3)$ & $12(0,7)$ \\
\hline Nootrópico & $0(0,0)$ & $0(0,0)$ & $2(0,4)$ & $2(0,4)$ & $4(0,2)$ \\
\hline Subtotal & $32(11,0)$ & $35(10,2)$ & $55(10,5)$ & $74(13,4)$ & $196(11,3)$ \\
\hline \multicolumn{6}{|l|}{ Sistema digestivo e metabolismo } \\
\hline Insulinas e outros agentes antidiabéticos & $11(3,8)$ & $10(2,9)$ & $33(6,1)$ & $33(7,0)$ & $87(5,0)$ \\
\hline Anti-secretor e antiácido & $16(5,5)$ & $16(4,6)$ & $24(4,5)$ & $20(3,6)$ & $76(4,4)$ \\
\hline Antiemético & $1(0,3)$ & $4(1,2)$ & $12(2,2)$ & $13(2,3)$ & $30(1,7)$ \\
\hline Hormônio tireoidiano/antitireoidiano e adjuvante & $3(1,0)$ & $8(2,3)$ & $14(2,6)$ & $8(1,4)$ & $33(1,9)$ \\
\hline Hipolipemiante & $2(0,7)$ & $9(2,6)$ & $11(2,0)$ & $8(1,4)$ & $30(1,7)$ \\
\hline Antiosteoporose & $0(0,0)$ & $1(0,3)$ & $0(0,0)$ & $1(0,2)$ & $2(0,1)$ \\
\hline Laxativo & $0(0,0)$ & $1(0,3)$ & $0(0,0)$ & $0(0,0)$ & $1(0,05)$ \\
\hline Antidiarréico & $0(0,0)$ & $0(0,0)$ & $2(0,4)$ & $2(0,4)$ & $4(0,2)$ \\
\hline Subtotal & $33(11,3)$ & $49(14,2)$ & $96(17,8)$ & $85(16,3)$ & $263(15,1)$ \\
\hline \multicolumn{6}{|l|}{ Uso sistêmico } \\
\hline Antiinflamatório não esteróide & $25(8,7)$ & $14(4,1)$ & $32(6,0)$ & $39(7,0)$ & $110(6,4)$ \\
\hline Analgésico & $35(12,1)$ & $28(8,1)$ & $26(4,8)$ & $40(7,1)$ & $129(7,5)$ \\
\hline Antiespasmódico & $1(0,3)$ & $0(0,0)$ & $2(0,4)$ & $8(1,4)$ & $11(0,6)$ \\
\hline Antiinflamatório esteróide & $7(2,4)$ & $5(1,4)$ & $34(6,3)$ & $10(1,8)$ & $56(3,2)$ \\
\hline Antibiótico & $19(6,6)$ & $12(3,5)$ & $29(5,4)$ & $26(4,6)$ & $86(5,0)$ \\
\hline Antifúngico & $1(0,3)$ & $8(2,3)$ & $4(0,7)$ & $2(0,4)$ & $15(0,9)$ \\
\hline Antiviral & $0(0,0)$ & $0(0,0)$ & $2(0,4)$ & $0(0,0)$ & $2(0,1)$ \\
\hline Antialérgico & $5(1,7)$ & $8(2,3)$ & $9(1,7)$ & $11(2,0)$ & $33(1,9)$ \\
\hline Detergente/Emoliente & $0(0,0)$ & $2(0,6)$ & $6(2,0)$ & $1(0,2)$ & $9(0,5)$ \\
\hline Subtotal & $83(32,1)$ & $77(22,3)$ & $144(27,7)$ & $137(24,5)$ & $451(26,1)$ \\
\hline
\end{tabular}

(continua) 
Tabela 4 (continuação)

\begin{tabular}{|c|c|c|c|c|c|}
\hline \multirow[t]{3}{*}{ Classes de medicamentos } & \multicolumn{5}{|c|}{ USF } \\
\hline & A & B & C & $\mathrm{D}$ & Total \\
\hline & n (\%) & $n(\%)$ & $n(\%)$ & $n(\%)$ & $n(\%)$ \\
\hline \multicolumn{6}{|l|}{ Sistema hematopoiético } \\
\hline Antiplaquetário & $9(3,1)$ & $17(4,9)$ & $17(3,2)$ & $27(4,8)$ & $70(4,1)$ \\
\hline Anticoagulante & $0(0,0)$ & $0(0,0)$ & $2(0,4)$ & $0(0,0)$ & $2(0,1)$ \\
\hline Antianêmico & $3(1,0)$ & $2(0,6)$ & $0(0,0)$ & $1(0,2)$ & $6(0,3)$ \\
\hline Venotônico e vasculoprotetor & $1(0,3)$ & $1(0,3)$ & $2(0,4)$ & $0(0,0)$ & $4(0,2)$ \\
\hline Subtotal & $13(4,4)$ & $20(5,8)$ & $21(3,9)$ & $28(5,0)$ & $82(4,7)$ \\
\hline \multicolumn{6}{|l|}{ Sistema respiratório } \\
\hline Broncodilatador & $6(2,1)$ & $16(4,6)$ & $12(2,2)$ & $27(4,8)$ & $61(3,6)$ \\
\hline Mucolítico/Fluidificante & $0(0,0)$ & $3(0,9)$ & $3(0,6)$ & $2(0,4)$ & $8(0,4)$ \\
\hline Descongestionante nasal & $0(0,0)$ & $0(0,0)$ & $0(0,0)$ & $0(0,0)$ & $0(0,0)$ \\
\hline Subtotal & $6(2,1)$ & $19(5,4)$ & $15(2,8)$ & $29(5,2)$ & $69(4,0)$ \\
\hline \multicolumn{6}{|l|}{ Fitoterápicos } \\
\hline Ginkgo biloba & $4(1,4)$ & $6(1,7)$ & $26(4,8)$ & $12(2,1)$ & $48(2,8)$ \\
\hline Castanha da Índia & $6(2,1)$ & $4(1,2)$ & $3(0,6)$ & $2(0,4)$ & $15(0,8)$ \\
\hline Cáscara sagrada & $0(0,0)$ & $0(0,0)$ & $0(0,0)$ & $0(0,0)$ & $0(0,0)$ \\
\hline Xarope de guaco & $2(0,7)$ & $6(1,7)$ & $5(0,9)$ & $6(1,1)$ & $19(1,1)$ \\
\hline Própolis e mel & $0(0,0)$ & $0(0,0)$ & $3(0,6)$ & $0(0,0)$ & $3(0,2)$ \\
\hline Outros fitoterápicos & $0(0,0)$ & $0(0,0)$ & $0(0,0)$ & $2(0,4)$ & $2(0,1)$ \\
\hline Subtotal & $12(4,2)$ & $16(4,6)$ & $37(6,9)$ & $22(4,0)$ & $87(5,0)$ \\
\hline \multicolumn{6}{|l|}{ Outros } \\
\hline Vitaminas e suplemento nutricional & $7(2,4)$ & $8(2,3)$ & $28(5,2)$ & $14(2,5)$ & $57(3,3)$ \\
\hline Antiparasitário & $8(2,8)$ & $13(3,8)$ & $4(0,7)$ & $7(1,3)$ & $32(1,8)$ \\
\hline Antifisético & $1(0,3)$ & $2(0,6)$ & $2(0,4)$ & $0(0,0)$ & $5(0,3)$ \\
\hline Anticalvície & $0(0,0)$ & $0(0,0)$ & $0(0,0)$ & $1(0,2)$ & $1(0,05)$ \\
\hline Antineoplásico & $1(0,3)$ & $0(0,0)$ & $0(0,0)$ & $0(0,0)$ & $1(0,05)$ \\
\hline Relaxante muscular & $0(0,0)$ & $0(0,0)$ & $1(0,2)$ & $0(0,0)$ & $1(0,05)$ \\
\hline Disfunção erétil & $0(0,0)$ & $0(0,0)$ & $1(0,2)$ & $0(0,0)$ & $1(0,05)$ \\
\hline Solução oftálmica lubrificante & $0(0,0)$ & $1(0,3)$ & $0(0,0)$ & $0(0,0)$ & $1(0,05)$ \\
\hline Subtotal & $17(5,8)$ & $24(7,0)$ & $36(6,7)$ & $22(4,0)$ & $99(5,7)$ \\
\hline Total & $289(16,7)$ & $345(19,9)$ & $537(31,0)$ & $560(32,4)$ & $1.731(100,0)$ \\
\hline
\end{tabular}

\section{Discussão}

Os dados do presente estudo possibilitaram a caracterização dos medicamentos prescritos aos idosos das quatro USF, quanto aos indicadores de prescrição medicamentosa propostos pela OMS, as classes farmacológicas, bem como a comparação do padrão de prescrição entre as USF.

No entanto, tais dados certamente não retratam a totalidade dos medicamentos utilizados pelos idosos, uma vez que não se considera a automedicação e medicamentos prescritos por outros médicos. Aponta-se, ainda, como limitação do estudo, a dificuldade de se obterem dados referentes a variáveis sócio-demográficas importantes quando se trata de caracterizar o uso de medicamentos, como raça, escolaridade, procedência e diagnóstico médico, devido à falta des- ses dados na maioria dos prontuários verificados nas USF.

Como esses dados foram coletados nos prontuários, e não nas receitas fornecidas aos idosos ao final da consulta, também não se pode afirmar que nas receitas constava o nome genérico do medicamento. Ou ainda, como nem sempre os medicamentos padronizados são fornecidos pelo município, o idoso pode ter adquirido o medicamento pelo nome comercial ou pelo nome genérico.

No que se refere à idade e ao sexo dos idosos estudados, os dados apontam para a maior freqüência das mulheres nas quatro unidades, representando $58,6 \%$ da população estudada, assim como em outros estudos 17. Todavia, quando comparadas aos homens da mesma idade, são consideradas mais vulneráveis às alterações no estado de saúde, tais como quedas, múltiplas 
doenças, obesidade, pobreza, dependências diversas e, conseqüentemente, ao uso de múltiplos medicamentos 18 .

O número de consultas por idoso ao ano, importante indicador de saúde para o planejamento das ações em saúde, mostrou que, nas unidades estudadas, a média geral foi de 3,8 consultas/idoso/ano, valor maior que o recomendado pelo Ministério da Saúde que é de 1,5 consultas/habitante/ano 19. Entretanto, estudo que avaliou fatores individuais associados à utilização de consultas médicas por adultos apontou que portadores de hipertensão arterial e diabetes mellitus têm maior probabilidade de realizar consultas 20 . Ao se considerar que tais doenças são freqüentes entre os idosos, pode-se pressupor que procurem consulta médica em número maior do que o preconizado. Além disso, os próprios protocolos de manejo dessas doenças recomendam consulta médica periódica. Fatores individuais, tais como sexo feminino, hospitalização no ano anterior, ex-tabagismo, e aumento da idade também tendem a aumentar o número de consultas 20 .

Ao se verificar o número de medicamentos prescritos por consulta médica foi constatado que, na USF A, em 71 (21,5\%) consultas, não foram prescritos medicamentos, havendo uma diferença significativa quando se compara com a USF B, em que não houve prescrição em apenas $20(7,6 \%)$ consultas médicas. Além disso, na USF A foram prescritos cinco ou mais medicamentos em $3 \%$ das consultas, enquanto na B essa ocorrência deu-se em $21,6 \%$ das prescrições. Em três USF (A, C e D), foi observado predomínio da prescrição de um único medicamento por consulta. Acrescenta-se que foram prescritos três medicamentos ou mais em $20,5 \%, 47 \%, 38,7 \%$, $41,9 \%$ das consultas, respectivamente, nas USF A, B, C e D. Embora a OMS proponha como padrão a prescrição de 1,3 a 2,2 medicamentos por consulta médica, tal critério não diferencia as faixas etárias, o que pode torná-lo insuficiente na comparação com os dados da população idosa, visto que ela utiliza com maior freqüência os serviços de saúde e também maior quantidade de medicamentos.

No estudo de Flores \& Mengue 21 , verificouse um consumo médio de medicamentos de 3,2 medicamentos por idoso, e $27 \%$ dos idosos apresentaram polifarmácia, ou seja, consumo superior a cinco medicamentos por pessoa. Monsegui et al. 11 e Sayd et al. 22 constataram o número médio de medicamentos usados pelos idosos entre dois e cinco. No estudo de Teixeira \& Lefévre 23 encontraram a prescrição de 3,6 medicamentos por idoso, outros estudos têm mostrado que $80 \%$ dos idosos tomam pelo menos um medicamento 24,25. Bertoldi et al. 26 constataram que $18 \%$ dos medicamentos prescritos a indivíduos acima de vinte anos são utilizados por idosos.

Mesmo que outros estudos também demonstrem que os idosos utilizam múltiplos medicamentos, hão de ser consideradas, no presente estudo, as diferenças nas características das prescrições medicamentosas que ocorrem entre as unidades, o que demanda outros estudos visando a caracterizar os motivos de tal ocorrência.

Referindo-se ao indicador apontado pela OMS e que orienta prescrição medicamentosa pela nomenclatura genérica das substâncias e/ ou princípios ativos que a compõem, conforme disposto na Resolução RDC no ${ }^{\circ} .135$, de 29 de maio de 200327 e na Resolução SS no. 64, de 30 de junho de $2004{ }^{28}$, no presente estudo o resultado foi de $91,7 \% ; 88,3 \% ; 87,2 \%$ e $85,7 \%$, respectivamente, nas USF A, B, C e D. Tais dados, apesar de não estarem em consonância com a meta de $100 \%$, podem representar um avanço quando comparados com os de outros estudos.

Santos \& Nitrini 29 , em estudo sobre indicadores de medicamentos em unidade básica de saúde, demonstraram uma freqüência de 30,6\% de medicamentos prescritos pelo nome genérico, dado semelhante ao evidenciado no estudo de Carneiro et al. 30, vale dizer, 33,9\%. Dados mais próximos aos encontrados neste estudo foram $71 \% 31,73,2 \% 32$ e $84,3 \% 33$.

Quanto às prescrições dos medicamentos para os idosos estudados, quando comparadas à lista de medicamentos selecionados pela Secretaria Municipal de Saúde de Marília, isto é, medicamentos padronizados como essenciais, foi constatado que $90 \%$ na USF A; $87,1 \%$ na USF B; $77,9 \%$ na USF C e de $77 \%$ na USF D estavam em consonância com ela e, portanto, com a Política Nacional de Medicamentos, fato que constitui um importante instrumento de orientação das ações de saúde relacionado ao uso de medicamentos, já que, por meio dessa orientação, é possível garantir a necessária segurança, eficácia e qualidade dos medicamentos, a promoção do uso racional e o acesso da população àqueles considerados essenciais 12 .

Neste aspecto, embora as USF C e D apresentem freqüências menores do que as demais, elas ainda estão dentro do padrão recomendado pela OMS, que é de, pelo menos, $70 \%$. Ademais, tais dados aparecem em freqüências maiores quando comparados com os do estudo de Carneiro et al. 30 que foram de 54,1\%. Em outros estudos foram encontradas freqüências que variaram de $82,4 \%$ a $87 \%$, dados que podem ser considerados mais próximos aos achados no presente estudo 29,31,32,34. 
Nas quatro unidades estudadas, o número de antibióticos por prescrição estava em conformidade com a orientação da OMS, que preconiza que seja inferior a $20 \%$ do total de medicamentos prescritos ao final da consulta, sendo a USF C aquela com maior freqüência de prescrição de antibiótico por consulta (10,1\%). Mesmo assim, chama a atenção o fato de na USF C ter sido prescrito o dobro de antibióticos comparativamente às demais USF $(5,1 \%$ na $A ; 4,5 \%$ na $B$ e $5,3 \%$ na D).

Quanto aos injetáveis, cuja recomendação é que corresponda até $10 \%$ dos medicamentos prescritos, foi encontrada a freqüência de 3,7\% na USF D e, nas demais, percentuais inferiores a esse.

As classes medicamentosas mais prescritas aos idosos têm sido aquelas que atuam no sistema cardiovascular, sendo $28,7 \%, 30,5 \%, 24,8 \%$ e $29,2 \%$, respectivamente nas unidades A, B, C e $\mathrm{D}$, com uma média de $27,9 \%$ do total de medicamentos prescritos aos idosos cujos prontuários foram verificados, sendo os hipotensores e diuréticos (15,2\% e 10,7\%, respectivamente) os mais prescritos. Dados semelhantes foram encontrados por Almeida et al. 35: $32 \%$.

Em estudo acerca do consumo de medicamentos com idosos acima de setenta anos foi demonstrado que 33,4\% dos grupos terapêuticos mais consumidos são os relacionados ao sistema cardiovascular 36 . No estudo de Coutinho \& Silva $37,54,7 \%$ dos idosos usavam anti-hipertensivos, e os diuréticos representavam $32,2 \%$ deles, mostrando que as drogas que atuam no sistema cardiovascular têm alta prescrição entre os idosos.

Quanto aos digitálicos, apenas 1,2\% dos medicamentos prescritos aos idosos deste estudo pertenciam a tal classe. Em estudo com idosos que vivem em comunidade foi encontrada uma freqüência de $6 \%$ naqueles com idade de 65 anos ou mais, e $9,4 \%$ com 75 anos ou mais 38 .

Medicamentos que atuam no metabolismo e sistema digestivo foram utilizados, conforme essa pesquisa, em 15,1\% dos idosos, enquanto na de Flores \& Mengue 21 foram prescritos para $18 \%$ dos idosos.

Os fármacos que atuam no sistema nervoso representaram neste estudo um percentual de $11,3 \%$, dados menores do que os encontrados por Flores \& Mengue 21, que constataram uma proporção de $22 \%$, e Coutinho \& Silva 37 , de $17,9 \%$. Neste último, os benzodiazepínicos eram utilizados por $15,1 \%$ e, no presente estudo, aponta-se um valor de $3,5 \%$.

Os antiagregantes plaquetários, neste estudo, atingiram o percentual de $4,1 \%$, bem abaixo dos $24,5 \%$ prescritos aos idosos na pesquisa de Coutinho \& Silva 37
Já os medicamentos de uso sistêmico, como os antiinflamatórios não esteróides, analgésicos e antibióticos, foram prescritos em $26,1 \%$ das consultas, constituindo o segundo grupo de medicamentos mais prescritos. Tal condição pode ser justificada pela alta ocorrência de dor em idosos, freqüentemente associada a desordens crônicas, particularmente às doenças músculoesqueléticas como artrites e osteoporoses 39 .

\section{Considerações finais}

Ao se utilizarem os indicadores da OMS como parâmetro para a comparação das prescrições medicamentosas em quatro USF da cidade de Marília, foi possível constatar a existência de uma tendência na busca de adequar as prescrições de acordo com os critérios indicados em relação ao número de medicamentos prescritos por consulta médica; número de consultas em que foram prescritos antibióticos; número de consultas em que foi prescrito pelo menos um injetável e número de medicamentos prescritos que figuram na lista de medicamentos padronizados pelo município.

Mesmo assim, considera-se a necessidade de avanços para serem atingidos melhores índices, sobretudo no que se refere ao uso do nome genérico dos medicamentos nas prescrições. A prescrição pelo nome genérico facilita a captação de informações a respeito dos fármacos na literatura internacional, pois sua nomenclatura não se modifica de acordo com interesses econômicos, como pode acontecer com os nomes comerciais e, finalmente, permite ao paciente a compra de um medicamento de menor custo.

Além disso, devem-se considerar as diferenças dos dados referentes aos indicadores de prescrições medicamentosas existentes entre as unidades estudadas. Pode-se imaginar que seja conseqüência do perfil do prescritor, já que o perfil de morbidade entre os idosos é semelhante, assim como as características das classes medicamentosas, segundo agrupamento anatômico demonstrado na Tabela 4.

Dessa forma, acrescenta-se a necessidade de estudos mais aprofundados que permitam maior exploração das variáveis estudadas e a caracterização de outros aspectos importantes como a correlação entre os medicamentos prescritos e as respectivas patologias presentes entre os idosos.

Acredita-se que os dados do presente estudo contribuam para despertar nos gestores e nos profissionais da saúde, a necessidade de constante busca de adequação e consenso no uso de medicamentos entre a população idosa. 


\section{Resumo}

Considerando que os idosos são grandes consumidores de medicamentos, com conseqüências significativas em sua condição de vida, propomos para o presente estudo analisar as características da prescrição de medicamentos, utilizando os indicadores propostos pela Organização Mundial da Saúde (OMS) e caracterizar as classes medicamentosas prescritas a eles, em quatro unidades da Estratégia Saúde da Família (ESF) de Marília, São Paulo, Brasil. Foi sorteada uma unidade de saúde da família (USF) por região da cidade e verificaram-se 100 prontuários na USF A; 86 na B, 99 na C e 97 na D. Quanto aos indicadores de prescrição de medicamentos da OMS, os resultados apresentam semelhanças com os padrões recomendados, exceto quanto à prescrição de medicamentos pelo nome genérico. Além disso, há diferenças significativas entre as unidades. As classes medicamentosas mais prescritas são aquelas destinadas aos problemas do sistema cardiocirculatório. Revela-se preocupação dos profissionais em adequar a prescrição de medicamentos a critérios de uso racional. Há, no entanto, necessidade de compreensão das diferenças de prescrição entre as unidades e busca de consenso, visando ao uso racional de medicamentos.

Prescrição de Medicamentos; Saúde do Idoso; Programa Saúde da Família

\section{Referências}

1. Terceira idade: dados estatísticos sobre os idosos. http://www.saudeemmovimento.com.br/conteu dos/conteudo_exibe1.asp?cod_noticia=91. (acessado em 26/Jun/2005).

2. Organização Mundial da Saúde. Envelhecimento ativo: uma política de saúde. Brasília: Organização Mundial da Saúde; 2005.

3. Lima-Costa MF. Epidemiologia do envelhecimento no Brasil. In: Rouquayrol MA, Almeida Filho N, organizadores. Epidemiologia \& saúde. 6a Ed. Rio de Janeiro: Editora Medsi; 2003. p. 499-513.

4. Kennedy DL, Forbes MB, Jones JK. Drug use in the United States in 1981. JAMA 1984; 251:1293-7.

5. Piraino AJ. Managing medication in the elderly. Hosp Pract (Minneap) 1995; 30:59-64.

6. Stolley JM, Buckwalter KC, Fjordbak B, Bush S. Iatrogenisis in the elderly: drug-related problems. J Gerontol Nurs 1991; 17:12-7.

7. Okuno J, Yanagi H, Tomura S, Oka M, Hara S, Hirano C, et al. Compliance and medication knowledge among elderly Japanese home-care recipients. Eur J Clin Pharmacol 1999; 55:145-9.

\section{Colaboradores}

C. A. P. Oliveira participou da elaboração do projeto, coleta, tabulação e análise dos dados e redação final. M. J. S. Marin orientou a elaboração do projeto, contribuiu na coleta, tabulação e análise dos dados e na redação final. M. Marchioli auxiliou na elaboração do projeto, incluindo a definição dos objetivos, delimitação metodológica, orientação da coleta, tabulação e análise dos dados e redação final. B. H. M. Pizoletto e R. V. Santos participaram da revisão da literatura, elaboração do projeto incluindo a definição dos objetivos e definição metodológica, além da coleta, tabulação, análise dos dados e redação final.

\section{Agradecimentos}

À Fundação de Amparo à Pesquisa do Estado de São Paulo (FAPESP) pelo financiamento do projeto de iniciação científica.
8. Lima-Costa MF, Barreto SM, Giatti L. Condições de saúde, capacidade funcional, uso de serviços de saúde e gastos com medicamentos da população idosa brasileira: um estudo descritivo baseado na Pesquisa Nacional por Amostra de Domicílios. Cad Saúde Pública 2003; 19:735-43.

9. Ramos LR. Os fármacos e o idoso. In: Gorzoni ML, Toniolo Neto J, organizadores. Terapêutica clínica no idoso. São Paulo: Editora Sarvier; 1995. p. 3-7.

10. Rozenfeld S. Prevalência, fatores associados e mau uso de medicamentos entre idosos: uma revisão. Cad Saúde Pública 2003; 19:717-24.

11. Mosegui GBG, Rozenfeld S, Veras RP, Vianna CMM. Avaliação da qualidade do uso de medicamentos em idosos. Rev Saúde Pública 1999; 33:437-44.

12. Ministério da Saúde. Portaria $\mathrm{n}^{\circ}$. 3.916, de 30 de outubro de 1998. Aprova a política nacional de medicamentos. Diário Oficial da União 1998; 10 nov. 
13. World Health Organization. The safety of medicines in public health programmes: pharmacovigilance na essential tool. Geneva: World Health Organization; 2006.

14. Barbetta PA. Estatística aplicada às ciências sociais. 3a Ed. Florianópolis: Editora dsa Universidade Federal de Santa Catarina; 1999.

15. Secretaria Municipal da Saúde de Marília. Relação de medicamentos padronizados. Marília: Secretaria Municipal de Saúde Marília; 2006

16. Melo JMS. DEF 2005/06: dicionário de especialidades farmacêuticas. 34a Ed. Rio de Janeiro: Epuc; 2005.

17. Fundação Sistema Estadual de Análise de Dados. Esperança de vida aumenta e diferença entre gêneros diminui: queda de homicídios em jovens poupa vidas e explica avanço masculino. São Paulo: Fundação Sistema Estadual de Análise de Dados; 2007.

18. Loyola Filho AI, Matos DL Giatti L, Afradique ME, Peixoto SV, Lima-Costa MF. Causas de internações hospitalares entre idosos brasileiros no âmbito do Sistema Único de Saúde. Epidemiol Serv Saúde 2004; 13:229-38.

19. Ministério da Saúde. Portaria no ${ }^{\circ}$ 91/GM, de 10 de janeiro de 2007. Regulamenta a unificação do processo de pactuação de indicadores e estabelece os indicadores do Pacto pela Saúde, a serem pactuados por municípios, estados e Distrito Federal. Diário Oficial da União 2007; 16 jan.

20. Capilhevia MF, Santos IS. Fatores individuais associados à utilização de consultas médicas por adulto. Rev Saúde Pública 2006; 40:436-43.

21. Flores LM, Mengue SS. Uso dos medicamentos por idosos em região do Sul do Brasil. Rev Saúde Pública 2005; 39:924-9.

22. Sayd JD, Figueiredo MC, Vaena MLHT. Automedicação na população idosa do núcleo de atenção ao idoso da UnATI-UERJ. Textos Envelhecimento 2000; 3:21-34.

23. Teixeira JJV, Lefèvre F. A prescrição medicamentosa sob a ótica do paciente idoso. Rev Saúde Pública 2001; 35:207-13.

24. Beyth RJ, Shorr RI. Epidemiology of adverse drug reactions in the elderly by drug class. Drugs Aging 1999; 14:231-9.

25. Monane M, Matthias DM, Nagle BA, Kelly MA. Improving prescribing patterns for the elderly through an online drug utilization review intervention: a system linking the physician, phamarcist, and compute. JAMA 1998; 280:1249-52.

26. Bertoldi AD, Barros AJD, Hallal PC, Lima RC. Utilização de medicamentos em adultos: prevalência e determinantes individuais. Rev Saúde Pública 2004; 38:228-38.

27. Agência Nacional de Vigilância Sanitária. Resolução RDC n ${ }^{\circ}$. 135, de 29 de maio de 2003. Aprova regulamento técnico para medicamentos genéricos. Diário Oficial da União 2003; 12 ago.
28. Secretaria Estadual de Saúde de São Paulo. Resolução SS nº. 64, de 30 de junho de 2003. Dispõe sobre a obrigatoriedade de prescrição de dispensação de medicamentos com o nome genérico das substâncias que os compõem. http://portal.saude.sp.gov. $\mathrm{br} /$ resources/geral/acoes_da_sessp/assistencia_ farmaceutica/resolucao_ss_64.pdf (acessado em 19/Fev/2008).

29. Santos V, Nitrini SMOO. Indicadores do uso de medicamentos prescritos e de assistência ao paciente de serviços de saúde. Rev Saúde Pública 2004; 38:819-26.

30. Carneiro RM, Marques MCP, Simões MJS. Estudo das prescrições de medicamentos em crianças de 0 a 2 anos atendidas no serviço municipal de saúde de Américo Brasiliense - SP, 1999. Rev Ciênc Farm 2000; 21:229-48.

31. Marcondes NSP. A assistência farmacêutica básica e o uso de medicamentos na zona urbana do município de Ponta Grossa, Paraná: um estudo de caso [Dissertação de Mestrado]. Rio de Janeiro: Escola Nacional de Saúde Pública, Fundação Oswaldo Cruz; 2002.

32. Naves JOS, Silver LD. Evaluation of pharmaceutical assistance public primary care in Brasília, Brazil. Rev Saúde Pública 2005; 39:223-30.

33. Cunha UGV, Barbosa MT, Paradela EM, Carvalho FG. Uso de digital em idosos admitidos em unidade de geriatria de hospital geral. Arq Bras Cardiol 1998; 71:695-8.

34. Colombo D, Santa Helena ET, Agostinho ACMG, Didjurgeit JSMA. Padrão de prescrição de medicamentos nas unidades de Programa de Saúde da Família de Blumenau. Rev Bras Ciênc Farm 2004; 40:549-58.

35. Almeida OP, Ratto L, Garrido R, Tamai S. Fatores preditores e conseqüências clínicas do uso de múltiplas medicações entre idosos atendidos em um serviço ambulatorial de saúde mental. Rev Bras Psiquiatr 1999; 21:152-7.

36. Gri E, Vázquez F, Barroso A, Cantero M, Monjo M, Juncosa S, et al. Consumo de medicamentos y remedios naturales en la población anciana de un área rural. Aten Primaria 1999; 23:455-60.

37. Coutinho ESF, Silva SD. Uso de medicamentos como fator de risco para fratura grave decorrente de queda em idosos. Cad Saúde Pública 2002; 18:1359-66.

38. Jain Y, Crome P. Digoxin in the elderly: a guide to therapy. Ger Med 1989; 19:35-8.

39. Andrade FA, Pereira LV, Sousa FAEF. Mensuração da dor no idoso: uma revisão. Rev Latinoam Enferm 2006; 14:271-6.

40. Secretaria Municipal de Saúde, Prefeitura Municipal de Marília. Sistema de informação da atenção básica - SIAB. Marília: Prefeitura Municipal de Marília; 2007.

Recebido em 11/Mar/2008

Versão final reapresentada em 29/Out/2008

Aprovado em 01/Dez/2008 